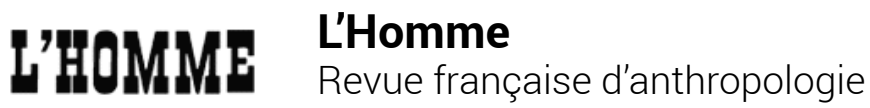

158-159 | avril-septembre 2001

Jazz et anthropologie

\section{Fran Markovitz, Coming of Age in Post-Soviet Russia}

Urbana \& Chicago, University of Illinois Press, 2000, XVII + 245 p., bibl., index, ph.

\section{Élisabeth Gessat-Anstett}

\section{OpenEdition}

Journals

Édition électronique

URL : http://journals.openedition.org/lhomme/6603

DOI : 10.4000//homme.6603

ISSN : 1953-8103

Éditeur

Éditions de l'EHESS

Édition imprimée

Date de publication : 1 janvier 2001

Pagination : 450-452

ISBN : 2-7132-1386-X

ISSN : 0439-4216

Référence électronique

Élisabeth Gessat-Anstett, «Fran Markovitz, Coming of Age in Post-Soviet Russia », L'Homme [En ligne], 158-159 | avril-septembre 2001, mis en ligne le 25 mai 2007, consulté le 24 septembre 2020. URL : http://journals.openedition.org/lhomme/6603 ; DOI : https://doi.org/10.4000//homme.6603

Ce document a été généré automatiquement le 24 septembre 2020.

(c) École des hautes études en sciences sociales 


\title{
Fran Markovitz, Coming of Age in Post-Soviet Russia
}

\author{
Urbana \& Chicago, University of Illinois Press, 2000, XVII + 245 p., bibl., \\ index, ph.
}

\author{
Élisabeth Gessat-Anstett
}

1 INFLUENCÉE par le courant culturaliste de l'anthropologie américaine, et plus particulièrement par les travaux de Ruth Benedict et de Margaret Mead, Fran Markovitz a cherché à appliquer une démarche méthodologique et théorique expérimentée depuis un demi-siècle dans le monde occidental à un vaste espace social récemment ouvert aux enquêtes qualitatives, à savoir la société postsoviétique. C'est en milieu urbain et sur leur lieu de scolarisation, à Moscou, et dans une moindre mesure à Saint-Pétersbourg et dans la ville provinciale de Dzerzinski, que l'auteur a réalisé des entretiens biographiques auprès d'une centaine de jeunes de seize ans au cours des hivers 1995 et 1996. L'anthropologue s'interroge en effet sur les conséquences des bouleversements politiques économiques et sociaux sur la vie des adolescents en Russie.

2 L'intérêt principal de l'ouvrage réside dans la richesse des données présentées, tant il est encore exceptionnel d'avoir accès à des sources directes d'information sur la société postsoviétique. La deuxième partie, proprement descriptive, restitue ainsi, à partir de nombreux extraits d'entretiens, le point de vue de jeunes Russes sur divers aspects de leur vie quotidienne. Mais l'intérêt de ce livre réside aussi dans la présentation des différentes facettes de la vie sociale adolescente, objet de la troisième partie consacrée à l'analyse des quatre grands sujets abordés dans les entretiens : les loisirs, la question de la nationalité, les identités sexuelles, religieuses et politiques, enfin les projets d'avenir.

3 Cependant, la lecture de Coming of Age in Post-Soviet Russia, par ailleurs très agréablement écrit, engendre chez le lecteur ethnologue une sorte d'inconfort qui trouve son origine dans un double problème d'ordre épistémologique. Le premier tient aux limites mêmes de l'étude. Certes, l'auteur revendique et argumente d'emblée (chap. II) le choix du cadre institutionnel dans lequel s'est déroulée son enquête. 
Toutefois, elle ne restitue que sporadiquement l'impact de cet environnemet si particulier et ne paraît guère prendre la mesure des contraintes que génère sur les pratiques de sociabilité adolescentes une institution dont les spécificités ont pourtant été étudiées par plusieurs historiens ${ }^{1}$. Il en va de même en ce qui concerne la société de référence. On a peine à identifier et à reconstituer l'écheveau des règles comportementales qui régissent la vie des adolescents. Peu de choses sont dites sur les catégories à partir desquelles la société russe se représente ce groupe d'âge et sur les normes qui président à leur insertion sociale. À cet égard, il est dommage que les travaux de l'ethnologue Tatiana Bernstam ${ }^{2}$ sur le rôle joué par les jeunes dans la vie rituelle de la société présoviétique n'aient pas servi de terme de comparaison à cette étude. Ils auraient permis à l'auteur de replacer son ethnographie dans le cadre d'ensemble de la culture russe.

4 Ce premier écueil, sur lequel achoppe l'ouvrage, me semble toutefois moins traduire la difficulté de travailler au sein d'une institution (des travaux sur les institutions sociales ou politiques des sociétés contemporaines ont été menés ailleurs avec succès) que sur celle propre à une approche morcelée du corps social dans le monde contemporain. Choisir d'enquêter exclusivement sur les adolescents en Europe est encore problématique, car le Léviathan moderne ne se laisse pas si facilement démembrer...

5 Le second problème que pose ce livre est plus épineux et relève de la méthode choisie. En effet, si la position d'anthropologue femme américaine d'une quarantaine d'année qui est celle de l'auteur est clairement présentée dès les premières pages, elle donne lieu à des assertions surprenantes de la part d'un chercheur qui revendique une approche ethnographique. Celle-ci avoue ainsi tout d'abord n'avoir été que "marginalement impliquée " dans la vie des adolescents interrogés (p.25), et finalement de ne pas « être en mesure de dire si leurs discours et leurs actes coïncident complètement » (p. 27), sans aller au-delà de ce simple constat.

6 Par ailleurs, il semble que Fran Markovitz ait mal mesuré l'incidence de son statut de chercheur étranger et de sa position d'observateur dans un univers (l'institution scolaire postsoviétique) extrêmement hiérarchisé où les rapports de pouvoir se traduisent parfois en termes de survie sociale et économique. Si l'on peut sourire en apprenant que l'ethnologue était véhiculée jusqu'à son lieu d'enquête dans la voiture et par le chauffeur du directeur régional du ministre de l'éducation, on regrette l'absence de réflexion épistémologique quant aux effets de cet encombrant parrainage sur le discours de ses informateurs.

7 De fait, au fur et à mesure de sa progression, l'ouvrage révèle une gênante directivité dont l'anthropologue n'a jamais pu se débarrasser au cours des entretiens. Le cadre analytique renvoie aux seuls choix de l'enquêtrice et ne semble révéler que partiellement l'univers de référence des enfants, souvent interloqués et déroutés par les questions posées. Les propos que l'auteur s'acharne à susciter (cf., p. 70, le paragraphe sur le «challenge ») prennent dès lors trop souvent la forme de paroles convenues. Devant l'uniformité d'un discours lisse, pauvre en émotions mais riche en bienséances et en bonnes intentions, le lecteur finit par douter que l'ethnologue américaine soit parvenue à recueillir et à restituer autre chose que la simple expression de la norme sociale.

8 L'auteur elle-même finit par s'étonner de tant de docilité et d'absence de rébellion de la part de jeunes adultes et en vient à conclure son enquête par une analyse de l'apprentissage, que feraient les adolescents, d'un pragmatisme social fait de doute et 
de cynisme, et motivé par une série de facteurs tant conjoncturels que structurels. Si bien que cette ethnologie des adolescents qui se cantonne par bien des aspects à une sociologie des bons élèves donne de façon ultime l'impression de rester à la surface des choses. Et celui qui, même brièvement, a eu la possibilité d'entrevoir la réalité de la société urbaine post-soviétique se demande si ce travail a été réalisé dans le même monde que celui qu'il a traversé, dans ce même Moscou où des centaines de besprizorniki (les enfants des rues) survivent d'expédients dans les égouts et les caves, et où chaque institution ou administration, qu'elle soit financière, scolaire, hospitalière ou carcérale, doit une part de sa survie à l'argent d'une corruption qui confère aux délinquants et aux criminels (mineurs ou majeurs) une impunité sans limite, dans un monde enfin où la cruauté et la violence des pauvres côtoie celle non moins folle et démesurée des riches, un monde bien différent en somme de l'univers aseptisé des enfants sages que Fran Markowitz a pu interroger.

\section{NOTES}

1. Cf. en particulier Wladimir Berelowitch, La Soviétisation de l'école russe, Lausanne, L'Âge d'Homme, 1990.

2. Tatiana Bernstam, Molodez-v ovbradonojzizni russkoj obsiny XIX-nacala XX v., Leningrad, Nauka, 1988.

\section{AUTEUR}

\section{ÉLISABETH GESSAT-ANSTETT}

Paris. 